\title{
Comprehensive Optimal Selection of Connection Mode for Distribution Network Based on FAHP
}

\author{
Jiacheng $\mathrm{Li}$ \\ North China Electric Power University, Beijing, 102206 \\ alanncepu@foxmail.com
}

Keywords: Distribution network; Fuzzy analytic hierarchy process (FAHP); IEEE-14 node system

\begin{abstract}
An optimal distribution connection mode can be worked out by use of Fuzzy Analytic Hierarchy Process (FAHP) to comprehensively evaluate connection modes in security, reliability and economy performances. We give a detailed step of FAHP algorithm, offering a new thought to evaluation of distribution network.
\end{abstract}

\section{Introduction}

With the rapid development of economy, the demand for electricity is growing, and requirements of reliability and economy performances in Electricity are more stringent. How to choose the optimal connection mode is an important piece of work in the planning and construction of MV power distribution network structure. The structure and connection of distribution network is complicated, and traditional mode only analyses the reliability and economy of it. The selection of connection is based on planners' personal experience and subjective judgment, which is unconvincing. Reference [1] proposes an integrated decision making method of connection mode for High voltage distribution network by use of Interval AHP.

This paper uses FAHP to evaluate different combinations of connection modes. The judgment matrix constructed in this paper can make full use of the information in alternative program, and can combine the qualitative and quantitative analysis organically. This provides new method to select optimal elementary connection mode for distribution network.

\section{The Theory of FAHP}

In order to adapt to the ambiguity and uncertainty of the judgment matrix, this paper uses the triangular fuzzy number proposed by Dutch scholar Van Laarhoven to give the fuzzy judgment matrix. It is expressed with an interval, instead of a single judgment value [2].

\section{Comprehensive Evaluation System for the Optimal Connection Mode Based on FAHP}

The Safety Evaluation Index. Safety indicators applying to all distribution network connection method are the fault severity index CSF and wiring safety index SS.

$$
C_{S F}=\left(\sum_{i=1}^{N_{B}} C_{F}^{i}\right) / N_{B}
$$

Where NB is the number of branches, $\mathrm{CFi}$ is a single fault severity index of the I line.

$$
S_{S}=k^{\prime} / C_{S F}
$$

Where $\mathrm{k}^{\prime}$ is the strength index of the spatial grid structure system. A distribution network connection security is higher, the larger the k' is, the probability of failure after load rejection will be smaller, the smaller the negative impact of the failure is.

The Reliability Evaluation Index. System mean power frequency index FSAIFI, system average outage duration index FSAIDI, the average power supply reliability index $\eta$ ASAI. 


$$
\begin{aligned}
F_{\text {SAIFI }}= & \frac{\sum_{i} \lambda_{s i} N_{i}}{\sum_{i} N_{i}} \\
F_{\text {SAIDI }}= & \frac{\sum_{i} N_{s i} N_{i}}{\sum_{i} N_{i}} \\
\eta_{\text {ASAI }}= & \frac{8760 \sum_{i} N_{i}-\sum u_{i} N_{i}}{8760 \sum_{i} N_{i}}
\end{aligned}
$$

Where $\lambda$ si is the average outage rate of load point $\mathrm{I}$; $\mathrm{Ui}$ is the average annual outage time; Ni is number of user.

The Economy Evaluation Index. Economic evaluation mainly includes two aspects of financial performance evaluation and technical and economic benefit evaluation. Financial evaluation is mainly from future load demand forecasting whether the investment will get profit; Technical and economic benefit evaluation is from the Angle of power distribution network itself to judge the economic benefits of planning scheme.

Financial Net Present Value Rate NPVR and Internal rate of return IRR are higher showing the better economic benefit.

$$
N_{P V R}=\frac{N_{P V}}{I_{p}}
$$

Where NPV is Net present value; IP is the present value of the investment; IRR is usually calculated by interpolation.

$$
I_{R R}=i_{1}+\frac{N_{P V}\left(i_{1}\right)}{N_{P V}\left(i_{1}\right)+\left|N_{P V}\left(i_{2}\right)\right|}\left(i_{2}-i_{1}\right)
$$

Where i1, i2 respectively taken by the trial with lower and higher discount rate; NPV (i1) NPV (i2) respectively was corresponding to the net present value of i1 and i2. Economic benefit evaluation of technology, the use of units of total assets of the maximum expected return as evaluation indexes FP:

$$
F_{P}=\frac{F_{\max }}{C_{E}}
$$

Where Fmax is grid maximum expected earnings; CE is total assets of grid. The index reflect the unit total assets of the maximum expected profit contribution of power grids, besides considering grid power supply capacity, also considering the power supply reliability, line loss, electricity price, the influence of such factors as the index. The higher the parameter values are, the better the power grid technology economy is.

Steps of FAHP Algorithm. Build Fuzzy Triangle Judgment Matrix. Pairwise comparing the indicators of each level, build the fuzzy triangle judgment matrix $A=($ aij) $n \times n$. It has the following characteristic:

(1) $a_{i i}=0.5, \quad i=1,2, \ldots, n$;

(2) $a_{i j}+a_{j i}=1, \quad i, j=1,2, \ldots, n$; 
In order to make any two solutions about the relative importance of certain standards for quantitative description, we usually use the 0.1 0.9 scaling, comparing a1, a2, .., an; we can get the following fuzzy triangle judgment matrix:

$$
A=\left[\begin{array}{llll}
a_{11} & a_{12} & \cdots & a_{1 n} \\
a_{21} & a_{22} & \cdots & a_{2 n} \\
\cdots & \cdots & \cdots & \cdots \\
a_{n 1} & a_{n 2} & \cdots & a_{n n}
\end{array}\right]
$$

Calculate the Weight of Fuzzy Triangle Judgment Matrix. Reference [3] inferred a formula calculating the weight of fuzzy triangle judgment matrix. The formula of fully contains the excellent characteristics of fuzzy triangle judgment matrix and its judgement information, small amount of calculation and convenient for computer programming implementation, has brought great convenience for practical applications.

$$
W_{i}=\frac{\sum_{j=1}^{n} a_{i j}+\frac{n}{2}-1}{n(n-1)}, i=1,2, \ldots, n
$$

Inspect the Consistency of Fuzzy Triangle Judgment Matrix. In order to judge if the result of weight is reasonable, reference [4] inferred a method of inspect the fuzzy triangle judgment matrix by using the compatibility of matrix:

Definition 1:

$$
I(A, B)=\frac{1}{n^{3}} \sum_{i=1}^{n} \sum_{j=1}^{n}\left|a_{i j}+b_{i j}-1\right|
$$

Where A, B are the index of compatibility, (aij)n $\times n$, (bij)n $\times n$ are fuzzy triangle judgment matrix Definition 2:

$\mathrm{W}=\left(\mathrm{W}_{1}, \mathrm{~W}_{2}, \ldots, \mathrm{W}_{\mathrm{n}}\right)^{\mathrm{T}}$ is the weight vector of $\mathrm{A}$,

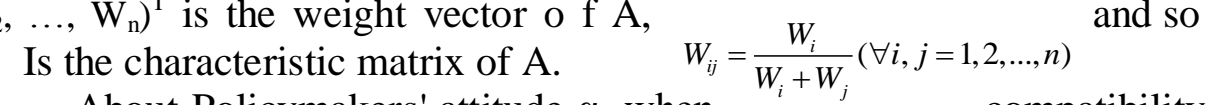

$W^{*}=\left(W_{i j}\right)_{n \times n} \quad$ About Policymakers' attitude $\alpha$, when $W_{i}+W_{j} \quad$ compatibility indicators $\mathrm{I}\left(\mathrm{A}, \mathrm{W}^{*}\right) \leq \alpha$, we could regard the judgement matrix as consistency. The smaller theais, which indicates policy makers on the consistency of fuzzy judgment matrix is higher, usually $\alpha=0.1$.

For the actual problem, experts give judgment matrix on the same factors $\mathrm{X}$.

$$
A_{k}=\left(a_{i j}^{(k)}\right)_{n \times n}, k=1,2, \cdots m
$$

They are all fuzzy triangle judgment matrix, so we can get their set of weights.

$$
W^{(k)}=\left(W_{1}^{(k)}, W_{2}^{(k)}, \cdots, W_{n}^{(k)}\right), k=1,2, \cdots, m
$$

Inspect satisfactory compatibility between the judgment matrixes.

$$
I\left(A_{k}, A_{l}\right) \leq \alpha, \quad k \neq l ; k, l=1,2, \cdots, m
$$

Then we take $\mathrm{m}$ set of weighted average as the weight of factors set $\mathrm{X}$ vector distribution.

$$
W_{i}=\frac{1}{n} \sum_{k=1}^{m} W_{i}^{(k)}, i=1,2, \cdots n
$$

Calculate Comprehensive Weight. After getting each layer of judgment matrix for local weight, then using the following formula to calculate connection method of a layer of attribute weights. 


$$
W_{i}^{k+1}=\sum_{j=1}^{n} W_{i j}^{(k)} W_{j}^{(k)}, i=1,2, \ldots, m
$$

Where m represents the number of connection method; ${ }^{W_{i}^{k+1}}$ is I connection method toward a certain attribute $A^{(k+1)}$ in $\mathrm{k}+1$ layer; $\mathrm{n}$ is the number of child attributes of ${ }^{(k+1)}$; is the weight of $W_{j}^{(k)} \quad A^{(k+1)}$

toward child attributes $\mathrm{j}$ in $\mathrm{k}$ layer; ${ }^{W_{i j}^{(k)}}$ is the weight of I connection method toward child attributes $\mathrm{j}$.

Finally, we select the highest weight connection mode, as a typical connection mode of distribution network planning.

\section{Conclusions}

This paper proposes a connection mode of distribution network based on elementary connection method. By use of Fuzzy Analytic Hierarchy Process (FAHP) this paper comprehensively evaluates the inferred elementary connection mode from these three aspects, reliability, economy and safety in qualitative and quantitative ways. Then the optimal connection mode for distribution network is achieved in this way, which avoids the inherent shortcomings of traditional connection modes, breaks the traditional analytical methods of distribution network, and makes the analysis or application of connection modes for distribution network easier.

\section{References}

[1] Xiao Jun, Wang Cheng-shan, Zhou Min, (2004) "AN IAHP-BASED MADM METHOD IN URBAN POWER SYSTEM PLANNING”, Proceedings of the CSEE, Vol.24, No. 4, pp51-57.

[2] Liu Zifa, Guo Huimeng \& LI Mengyu, (2014) "Study on Power Grid Blackout risk under Different Connection Modes", Electric power construction, Vol. 35, No.8, pp25-29.

[3] Liu Gui-Long, (2004) "The Axiomatic Systems of Rough Fuzzy Sets on Fuzzy Approximation Spaces”, CHINESE JOURNAL OF COMPUTERS, Vol. 27, No.9, pp1188-1191.

[4] CHEN Hua-you, Zhao Jia-bao, (2004) "Research on Compatibility of Fuzzy Judgement Matrices", OPERATIONS RESEARCHAND MANAGEMENT SCIENCE, Vol. 13, No.1, pp44-47.

[5] Ji dong-chao, Song bi-feng \& Yu tian-xiang, (2006) "FAHP and its application in the selection of design scheme", Systems Engineering and Electronics, Vol. 28, No. 11, pp160-166.

[6] Wang wei, Zhang li-zi \& Ma xiu fan, (2006) "Based on the theory of structural units of medium voltage distribution network connection mode". Automation of electric power system, Vol.30, No.11, pp35-39.

[7] Liu Xiang jun, MA Shuang \& XU Gang, (2012) "Formation of Typical Connection Mode for Distribution Network by Elementary Connection Model", Power System Technology, Vol.36, No. 2, pp59-63.

[8] Guo jinyu \& Zhang zhongshan \& Sun qingyun, "The research and application of analytic hierarchy process", Chinese journal of safety science, Vol.18, No.5, pp149-153.

[9] Chang jiane \& Jiang taili, "Analytic hierarchy process (ahp) to determine weights of research", Journal of wuhan university of technology, Vol.29, No.1, pp151-155.

[10] Si J D, Tang E T, Xu G. Safety research in typical connection models of distribution system[C]//Asia-Pacific Power and Energy Engineering Conference. Chengdu, China IEEE Power \& Energy Society (PES), 2010: 1-4. 
[11] Jia Z X, Zhang H B, Xi A M. Research in method of complex system reliability evaluation based-on fuzzy sets[C]//International Workshop on Intelligent Systems and Applications. Wuhan, China: Hubei University of Technology, 2009: 1-4. 